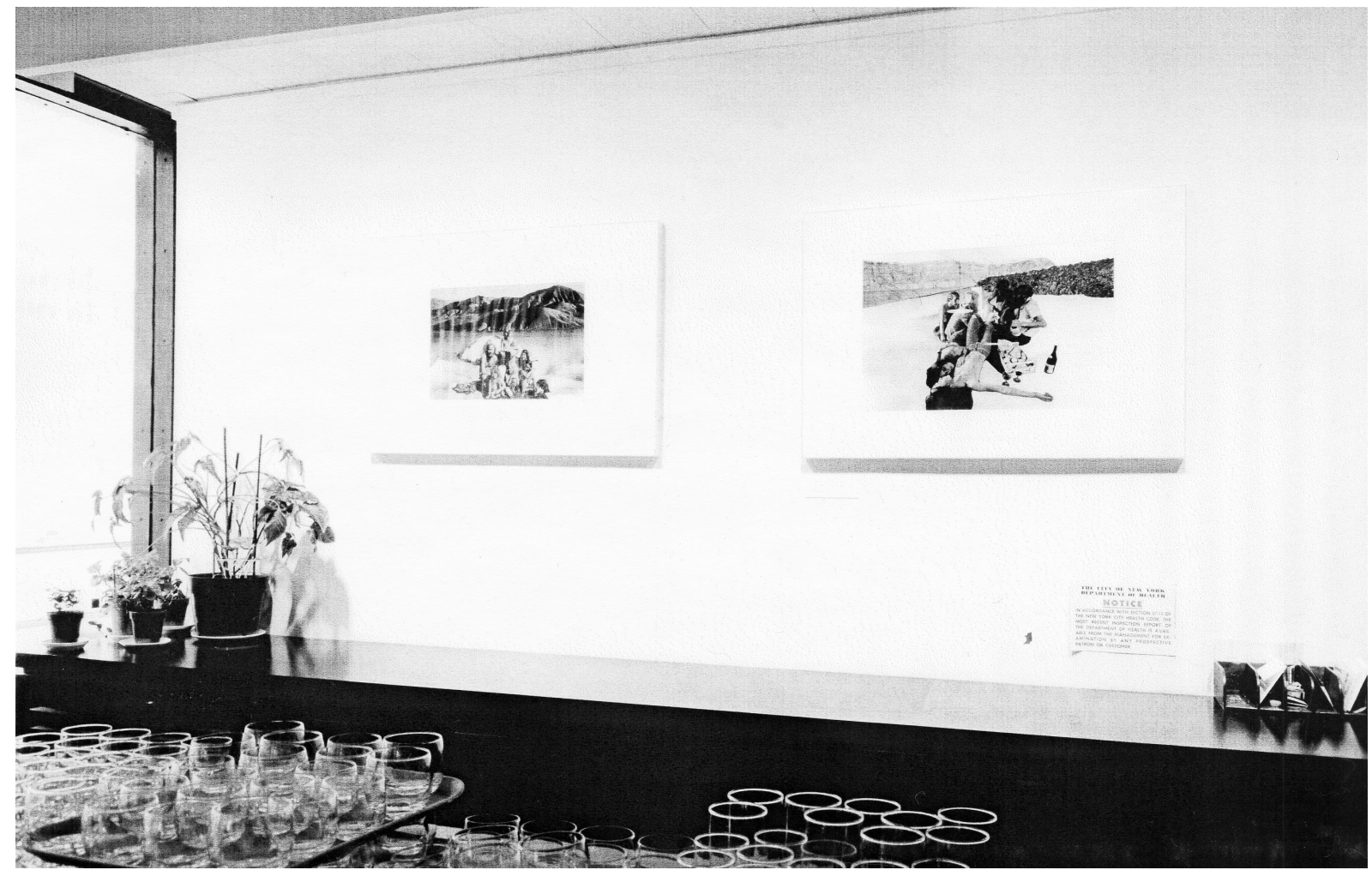




\section{Antecedentes: Cuando la arquitectura se hizo arte (1975-1977) Carlos Mínguez}

Los ejemplos recogidos en este escrito nos hablan de los primeros casos donde los documentos arquitectónicos, especialmente los dibujos, fueron absorbidos por el mercado del arte a través de las galerías comerciales de mediados de los años setenta en la ciudad de Nueva York'.

\section{PALABRAS CLAVE}

Arquitectura, comisariado, exposiciones, galerías, MoMA

\section{KEYWORDS}

Architecture, Curating, Exhibitions, Galleries, MoMA

A pesar de varias décadas de importantes exposiciones en el Museo de Arte Moderno (MoMA en sus siglas en inglés), fue relativamente raro que la arquitectura se exhibiera en las galerías de arte de Nueva York. Sin embargo, a finales de los años setenta, en el transcurso de escasos tres años, la ciudad comenzó a ver arquitectura en importantes galerías comerciales a través de sus dibujos, maquetas, e instalaciones. ¿Qué pasó durante estos años? ¿Cuál es la razón de tal aparición de exposiciones de arquitectura en tan poco tiempo?

Esta transformación se produjo en cierta medida por el aumento del interés alrededor de los dibujos de arquitectura, en parte precipitada por la importancia y repercusión de la exposición The architecture of the Ecole des Beaux-Arts organizada por Arthur Drexler en el MoMA, y abierta al público el 29 de octubre de 1975.

La exposición presentó principalmente el trabajo de estudiantes en acuarela a gran escala del siglo XIX de la escuela parisina, incluyendo también proyectos de Henri Labrouste, Charles Garnier y otros edificios franceses y estadounidenses del mismo estilo. La mayor parte de la exposición estuvo dedicada a la presentación de ejercicios de los alumnos. En otras palabras, la exposición presentó una colección de dibujos de edificios que nunca se dibujaron con el objetivo de ser construidos. Y algo más: eran bellos.

\section{Carlos Mínguez Carrasco}

Arquitecto y curador de arquitectura. Actualmente es Senior Curator de ArkDes, el Museo Nacional de Arquitectura y Diseño de Suecia. Fue Curador Asociado de Storefront for Art and Architecture entre 2012 y 2017. Anteriormente fue Curador en Jefe de la Trienal de Arquitectura de Oslo en 2016, junto a la After Belonging Agency, y Curador Asistente de OfficeUS, el pabellón de Estados Unidos en la Bienal de Arquitectura de Venecia de 2014. Graduado en la Escuela Superior de Arquitectura de Barcelona ETSAB, recibió el Máster en Crítica, Curadoría, y Prácticas Conceptuales en Arquitectura en Columbia University, Nueva York. Ha organizado un amplio rango de exposiciones, eventos y concursos con un particular interés alrededor de cómo las disputas sociales y políticas influencian la cultura arquitectónica contemporánea. Ha sido editor de varias publicaciones, incluyendo After Belonging: The Objects, Spaces, and Territories of the Ways We Stay in Transit (Lars Müller Publishers, 2016), OfficeUS Manual (Lars Müller Publishers, 2017) y Bodybuilding: Performance and Architecture (Performa, 2019) Sus textos han sido publicados en diversos catálogos de exposiciones y en revistas como Domus, Migrant Journal, o Código. Ha dado conferencias en diferentes universidades y espacios culturales de Europa, Estados Unidos y Latinoamérica, incluyendo Columbia University GSAPP, Harvard University GSD, y Princeton University SoA.

Fig. 01

Vista de la exposición Architectural Studies and Projects, Ático del Museo de Arte Moderno (MoMA), Nueva York, 1975. 
Como dijo Paul Goldberger en su reseña de la exposición en el New York Times:

\footnotetext{
"Visualmente, esta es la exposición arquitectónica más hermosa que me viene a la memoria, y se encuentra entre los espectáculos más atractivos de cualquier tipo jamás montados en Nueva York"2.
}

Así pues, aparte de ser un evento transformador para la disciplina de la arquitectura, la exposición presentó documentos de arquitectura que podían ser entendidos fácilmente por los no arquitectos, apreciándose únicamente por sus colores y belleza. Las espectaculares acuarelas y dibujos Ilamaron la atención especialmente por su estética, dejando de lado su uso, propósito, contexto, o la tecnología utilizada ${ }^{3}$.

Siete meses antes de la inauguración de la gran exposición sobre la Beaux-Arts, Emilio Ambasz, en ese momento curador de diseño en el MoMA, organizó una pequeña exposición llamada Architectural Studies and Projects (Estudios y proyectos de arquitectura).

La exposición se instaló en el ático del museo, y abrió sus puertas a partir del 13 de marzo hasta el 15 de mayo de 1975. El penthouse, situado en la sexta planta del MoMA incluía el espacio dedicado al servicio de préstamo de arte, también conocido como la galería de venta y alquiler del museo, que servía a su vez como salón y restaurante para los miembros del museo 4 .

El servicio de préstamo, en inglés Art Lending Service (ALS), era un departamento que brindaba a los miembros del museo la oportunidad de alquilar una obra de arte por un período de dos meses antes de decidir si comprar la obra o devolverla a la ALS. EI ALS percibía un porcentaje de la obra alquilada o vendida de una manera similar a como lo haría una galería comercial.

EI ALS comenzó a funcionar en 1951 como una forma de conectar el museo con las galerías y coleccionistas de Nueva York, produciendo exposiciones en el ático del museo desde 1955. Las primeras exposiciones consistieron en una selección de obras de la propia colección del ALS, pero desde principios de la década de los sesenta, las exposiciones comenzaron a ser un poco más elaboradas, debido a la creación del AAS, El Art Advisory Service. (El servicio de asesoramiento de arte). Apoyado por miembros del llamado "Consejo Junior" del museo, este servicio adjunto de la ALS fue fundado con la intención de desarrollar la colección de arte contemporáneo de la institución. Así pues, desde 1962, las exposiciones que se llevaron a cabo en el ático comenzaron a tener una base temática y la AAS comenzó a llamar a curadores invitados para organizar las muestras. Curadores como Campbell Wyly, Pierre Apraxine, o John Garrigan organizaron numerosas exposiciones, dedicadas principalmente a la pintura, la escultura o la fotografía con títulos como: Young West artists (1965), Nine print portfolios (1970), o Prints for collectors (1972).

La exposición titulada Architectural Studies and Projects en 1975 supuso la primera vez que el ALS organizaba una exposición de arquitectura, y también fue la primera vez que se invitaba a Emilio Ambasz como curador invitado.

Emilio Ambasz fue curador de diseño en el Museo de Arte Moderno desde 1970 hasta 1976. Organizó diversas exposiciones de arquitectura y diseño industrial, entre ellos Italy: The New Domestic 
Landscape, en 1972; The architecture of Luis Barragán, en 1974; y The Taxi Project, en 1976.

Architectural Studies and Projects presentó 52 dibujos de arquitectura, definidos como una serie de "proyectos visionarios, creaciones imaginarias nunca pensadas para construirse" según el propio Ambasz". La exposición fue una "exposición informal, la primera de una serie, que presenta trabajos recientes de arquitectos estadounidenses y europeos". Ambasz invitó a 23 arquitectos y grupos de arquitectos a presentar un máximo de tres trabajos en papel para la exposición.

Los dibujos expuestos en la muestra cubrieron una amplia gama de proyectos y estilos, reuniendo importantes representantes de las diferentes líneas de pensamiento arquitectónico del momento. Si los repasamos por países, los autores presentados fueron: Desde Austria: Raimund Abraham, y Friederick St. Florian; Italia: Ettore Stottsass, Superstudio, Gaetano Pesce, Lauretta Vinciarelli y Alessandro Mendini; Holanda: Eventstructures, Rem Koolhaas junto con Elia y Zoe Zenghelis: Gran Bretaña: Peter Cook, Michael Webb y Cedric Price; Estados Unidos: Peter Eisenman, Michael Graves, John Hedjuk, Richard Meier y SITE INC; Argentina: Rodolfo Machado y Susana Torre. Los precios de las obras oscilaron entre 200 y 2000 dólares. Como práctica habitual, el Servicio de Préstamo de Arte tenía una comisión del 15\% en todas las ventas.

Judith Prince, Presidenta de la ALS, al hablar de la exposición en una de las cartas de invitación, deja claro la intención comercial del programa:

\footnotetext{
"Esperamos que acepte participar en lo que creemos que será una exposición significativa para presentar al público coleccionista los dibujos de importantes arquitectos" 6 .
}

Si bien las diferencias ideológicas entre los proyectos presentados eran muy grandes, la recepción de la exposición los equiparó debido de sus características poéticas y visionarias. Paul Goldberger de nuevo, en su reseña en el New York Times dice:

\footnotetext{
"Architectural Studies and Projects, que se inauguró hoy en el ático del Museo de Arte Moderno, trata sobre el aspecto más periférico, pero quizás el más lujoso, de la arquitectura: la creación. Trata de esquemas de dibujos puramente visionarios que no tienen conexión con la realidad"”.
}

La exposición fue capaz de presentar los dibujos arquitectónicos como arma de seducción para el público en general, y para el mercado del arte en particular. Goldberger seguía: "Uno de los objetivos del espectáculo ha sido encontrar interés público en el dibujo arquitectónico como arte, y a este nivel es probable que tenga éxito"s.

Tuvo éxito: No sólo porque once de los 23 autores vendieron su trabajo, sino también porque los coleccionistas de arte ya habían mordido el anzuelo.

Pierre Apraxine trabajó como asistente curatorial de pintura y escultura para el Museo de Arte Moderno desde el verano de 1970 hasta 1973. En el momento de Architectural Studies and Projects, comenzó a trabajar como curador de Howard Gilman, propietario de la Gilman Paper Company con el objetivo de armar una colección de arte contemporáneo. La colección estaba pensada para ser construida alre- 
dedor de arte minimalista y conceptual, pero Pierre Apraxine, al visitar la exposición en el ático del MoMA cambió de opinión y decidió comenzar a coleccionar dibujos arquitectónicos, creando la llamada "Colección de Dibujos Visionarios Howard Gilman" que se inició en 1975 y estuvo casi terminada en 1980. La colección fue donada al Museo de Arte Moderno en noviembre del año 2000'.

En 1977, tres exposiciones de arquitectura abrieron sus puertas en galerías comerciales en Nueva York: Towards a More Modern Architecture (Hacia una arquitectura más moderna), organizada por Robert Stern e inaugurada en el Drawing Center y en el Cooper Hewitt Museum. New York: Past, Present, and Proposed (Nueva York: Pasado, presente y propuesto) en la recién inaugurada Gallery Spaced, y la más importante de las tres: Architecture I (Arquitectura I), curada por el propio Pierre Apraxine en la galería Leo Castelli.

Leo Castelli, conocida en ese momento como una de las galerías de arte más influyentes de Nueva York, estaba dedicada especialmente a movimientos artísticos como el expresionismo abstracto, el minimalismo y el arte pop, representando a artistas como Robert Rauschenberg, Jasper Johns, Frank Stella, o Cy Twombly. La exposición en Leo Castelli fue la más significativa de estas tres exposiciones, no tanto por el trabajo expuesto, sino porque reveló hasta qué punto los dibujos arquitectónicos se habían convertido en un fenómeno del mundo del arte. Tras el éxito de Architecture l, la galería ya en 1977 comenzó a representar a arquitectos y a organizar una serie de exposiciones de arquitectura durante los años siguientes, siguiendo con Architecture II: Houses for Sale (Arquitectura Il: Casas en venta) en 1980, o la tercera de la serie Architecture III: Follies (Arquitectura III: Locuras) en 1983.

La exposición en la galería Castelli presentó las obras de siete arquitectos: Raimund Abraham, Emilio Ambasz, Richard Meier, Walter Pichler, Aldo Rossi, James Stirling y la firma Venturi y Rauch. Tres arquitectos y el propio curador repetían con respecto a la exposición del ático del MoMA. La exposición, según su curador, buscó "ilustrar las diversas actitudes estéticas y filosóficas que prevalecen en la arquitectura contemporánea. Presenta, por lo tanto, una sección transversal, en lugar de una sola doctrina arquitectónica" ${ }^{\prime \prime}$.

Hubo muchas más exposiciones de arquitectura en galerías de arte durante los años posteriores. Algunas de ellas difirieron en contenido, formato, y en objetivo, pero la mayoría de ellas coinciden en una característica: La representación arquitectónica se programó, seleccionó, presentó, y promovió con el objetivo de ser vendida.

Las galerías comerciales vieron la oportunidad de vender obra que provenía de un ámbito sin explotar. Por otro lado, algunos arquitectos vieron en la galería un espacio para desarrollar ideas y proyectos que no tenían cabida en el mundo profesional, pero tampoco en el ámbito más disciplinario de las publicaciones o de las revistas de arquitectura. La galería comercial ofrecía un espacio complementario al resto de formatos de circulación de las ideas contemporáneas en arquitectura, situado entre la diseminación de las publicaciones y la consolidación histórica que ofrecían los museos.

La galería comercial ofrecía también una audiencia peculiar. Así como las revistas de arquitectura eran mayoritariamente leídas por una audiencia disciplinaria (desde estudiantes a académicos y profesionales de la arquitectura), la audiencia de las exposiciones de 
arquitectura en las galerías comerciales de Nueva York era un público mucho más heterogéneo, formado por expertos y profesionales, dealers, curadores y coleccionistas de arte, pero también por un público aficionado, expuesto a las tendencias artísticas más vanguardistas pero en su mayoría con poco o ningún conocimiento adelantado de arquitectura. Tampoco se trataba de la "audiencia" de un edificio construido, formado por los usuarios directos, los habitantes de una ciudad, o los receptores de los textos, documentos, fotografías y promociones de tal edificio.

Es importante advertir que el formato de presentación de los trabajos arquitectónicos por parte de las galerías comerciales fue marcadamente convencional. Los dibujos y maquetas son en su mayoría tratadas como si fueran obras de arte. Su presentación se hace sin ningún tipo de contexto, equiparando una planta de un proyecto de vivienda a una pintura, y una maqueta de un edificio público a una escultura. La presentación de los trabajos enfatizó el nombre del autor -reforzando la idea del arquitecto como artista- y no ofrecían ningún tipo de sofisticación en revelar los componentes sociales, políticos y culturales que los proyectos contenían.

La incorporación de las representaciones arquitectónicas en las salas de exposición de las galerías de arte más importantes de la ciudad de Nueva York no sólo es una evidencia del interés del mundo del arte hacia este material. También significa una tendencia de la práctica arquitectónica que vio en las salas de la galería de arte un nuevo solar donde construir tanto sus ideas como sus nombres y apellidos. RA

\section{Notas}

01. Esta investigación fue iniciada en 2009 en el ámbito del programa Critical, Curatorial, and Conceptual Practices in Architecture en Columbia University GSAPP, y desarrollada durante los años posteriores. Desde entonces han habido diferentes artículos y publicaciones sobre casos similares. El trabajo más completo que desarrolla y expande los casos de estudio perfilados en este texto es KAUFFMAN, Jordan, "Drawing on Architecture, The Object of Lines 19701990" MIT Press, 2018.

02. GOLDBERGER, Paul, "Beaux Art Architecture at the Modern "New York Times, 29 de octubre de 1975.

03. No sería la única intención de su curador, Arthur Drexler. Para información detallada de la exposición, su proyecto curatorial, y su repercusión en el discurso arquitectónico, se puede consultar Felicity D. Scott, "When Systems Fail", en Architecture and Techno-utopia, MIT Press, 2007.

04. PRICE, Judith, Presidente del Art Lending Service, en una carta a Andrea Branzi el 7 de Noviembre de 1974.

05. El Museo de Arte Moderno, Comunicado de Prensa Architectural Studies and Projects, 13 de marzo de 1975.

06. PRICE, Judith, Presidente del Art Lending Service, en una carta a Andrea Branzi el 7 de Noviembre de 1974.

07. GOLDBERGER, Paul, "Beaux Art Architecture at the Modern" New York Times, 29 de octubre de 1975.

08. Idem.

09. APRAXINE, Pierre, en conversación con Paola Antonelli, en el catálogo de la exposición The Changing of the AvantGarde: Visionary Architectural drawings from the Howard Gilman collection, Museo de Arte Moderno, 2002.

10. APRAXINE, Pierre, en el prólogo del catálogo Arquitectura I, Nueva York, 1977.
RA. Revista de Arquitectura Núm. 21 - 2019

P. $32-37$ 